\title{
NONLINEAR ASTEROSEISMOLOGY OF RR LYRAE
}

\author{
L. Molnár ${ }^{1}$, Z. Kolláth ${ }^{1}$, R. Szabó ${ }^{1}$, S. Bryson ${ }^{2}$, K. Kolenberg ${ }^{3,4}$, F. Mullally Kon and S. E. Thompson $^{2,5}$ \\ ${ }^{1}$ Konkoly Observatory, MTA CSFK, H-1121 Budapest, Konkoly Thege Miklós út 15-17, Hungary; molnar.laszlo@ csfk.mta.hu \\ 2 NASA Ames Research Center, MS 244-30, Moffet Field, CA 94035, USA \\ ${ }^{3}$ Harvard-Smithsonian Center for Astrophysics, 60 Garden Street, Cambridge MA 02138, USA \\ ${ }^{4}$ Institute of Astronomy, KU Leuven, Celestijnenlaan 200D, B-3001 Heverlee, Belgium \\ ${ }^{5}$ SETI Institute, 189 Bernardo Ave Suite 100, Mountain View, CA 94043, USA \\ Received 2012 July 17; accepted 2012 August 16; published 2012 September 4
}

\begin{abstract}
The observations of the Kepler Space Telescope revealed that fundamental-mode RR Lyrae stars may show various radial overtones. The presence of multiple radial modes may allow us to conduct nonlinear asteroseismology: comparison of mode amplitudes and frequency shifts between observations and models. Here we report the detection of three radial modes in the star RR Lyr, the eponym of the class, using the Kepler short cadence data: besides the fundamental mode, both the first and the ninth overtones can be derived from the data set. RR Lyrae shows period doubling, but switches occasionally to a state where a pattern of six pulsation cycles repeats instead of two. We found hydrodynamic models that show the same three modes and the period-six state, allowing for comparison with the observations.
\end{abstract}

Key words: stars: individual (RR Lyr) - stars: oscillations - stars: variables: RR Lyrae

Online-only material: color figures

\section{INTRODUCTION}

The precise and continuous observations of the Kepler Space Telescope (Borucki et al. 2010) revealed a wealth of new features in RR Lyrae stars. Among the first discoveries was the detection of period doubling in three stars, including RR Lyrae itself (Kolenberg et al. 2010; Szabó et al. 2010). Additional frequencies in the Fourier transform, significant frequency components besides the usual RR Lyrae pattern (main period, its harmonics, modulation sidelobes, and the Blazhko-modulation frequency), were also detected in several stars (Benkő et al. 2010; Guggenberger et al. 2012). Out of these new features, the period-doubling phenomenon, the alternation of a higher and a lower amplitude pulsation cycle, has been successfully modeled. Our hydrodynamic calculations revealed that a highorder, 9:2 resonance can occur between the fundamental mode and the ninth radial overtone strange mode that leads to the period-doubling bifurcation (Kolláth et al. 2011). These results were also confirmed by Smolec et al. (2011).

However, the nature of the additional frequencies is not fully understood yet, except for the ninth overtone which is related to the half-integer peaks and causes the period doubling. Some of the remaining peaks fall in the ranges where the first or second radial overtones are expected, but several stars display even more peaks, possibly indicating non-radial modes (Benkő et al. 2010; Guggenberger et al. 2012). Additional modes were also detected in stars observed by the CoRoT Space Telescope (Chadid et al. 2010; Poretti et al. 2010; Guggenberger et al. 2011). The stars showing first or second overtone signals are unlike the usual double-mode RRd pulsators. The additional components fall in the millimagnitude range, so the amplitude ratios are extreme.

Furthermore, almost all stars that show additional modes are Blazhko variables. Only two stars have been found in the Kepler sample that show a small second overtone with only traces of modulation down to the millimagnitude level (Benkő et al. 2010; Nemec et al. 2011). On the other hand, about half of the modulated stars in the Kepler sample show additional peaks in the Fourier spectrum, often together with signs of period doubling. Period doubling itself is not a regular process: It is modulated and sometimes shows signatures of additional bifurcations. The variations do not necessarily follow the Blazhko modulation either (Szabó et al. 2010). Therefore, it is necessary to investigate the origins of those variations.

\section{KEPLER PHOTOMETRY OF RR LYRAE}

RR Lyr (KIC 7198959) is one of the brightest stars observed by Kepler among its asteroseismology targets (Gilliland et al. 2010). It was observed in long cadence mode in Kepler quarters 1 and 2 (or Q1 and Q2), with 29.4 minute sampling. Kolenberg et al. (2011) provided a detailed description of the photometry and the necessary corrections applied. The brightness of RR Lyr was initially underestimated in the Kepler Input Catalog (KIC) and some flux was lost from the less-than-ideal aperture. However, the corrected average brightness resulted in an assigned automatic aperture that extended beyond the edge of the CCD module and so the pipeline rejected the star in quarters 3 and 4 . A custom aperture had to be developed for the star instead and it is being used from quarter 5 onward. RR Lyrae was observed through the Kepler Guest Observer Program ${ }^{6}$ in short cadence mode (1 minute sampling) both in Q5 and Q6.

Maxima of the pulsation cycles had to be determined for our analysis. For the long cadence data, we reused the maxima derived from ninth-order polynomials fitted in Szabó et al. (2010). For the short cadence data we calculated spline solutions instead of polynomials. Long cadence data are less well suited to determine the sharp maxima of RR Lyrae-type variations, but we found the results satisfactory for further analysis.

\subsection{Analysis of the Photometry}

In principle, return maps would be very useful to analyze the variations in period doubling detected in RR Lyrae stars. Return maps are powerful tools to examine the overall dynamics of a system by plotting the successive values of a selected property

\footnotetext{
6 http://keplergo.arc.nasa.gov/
} 


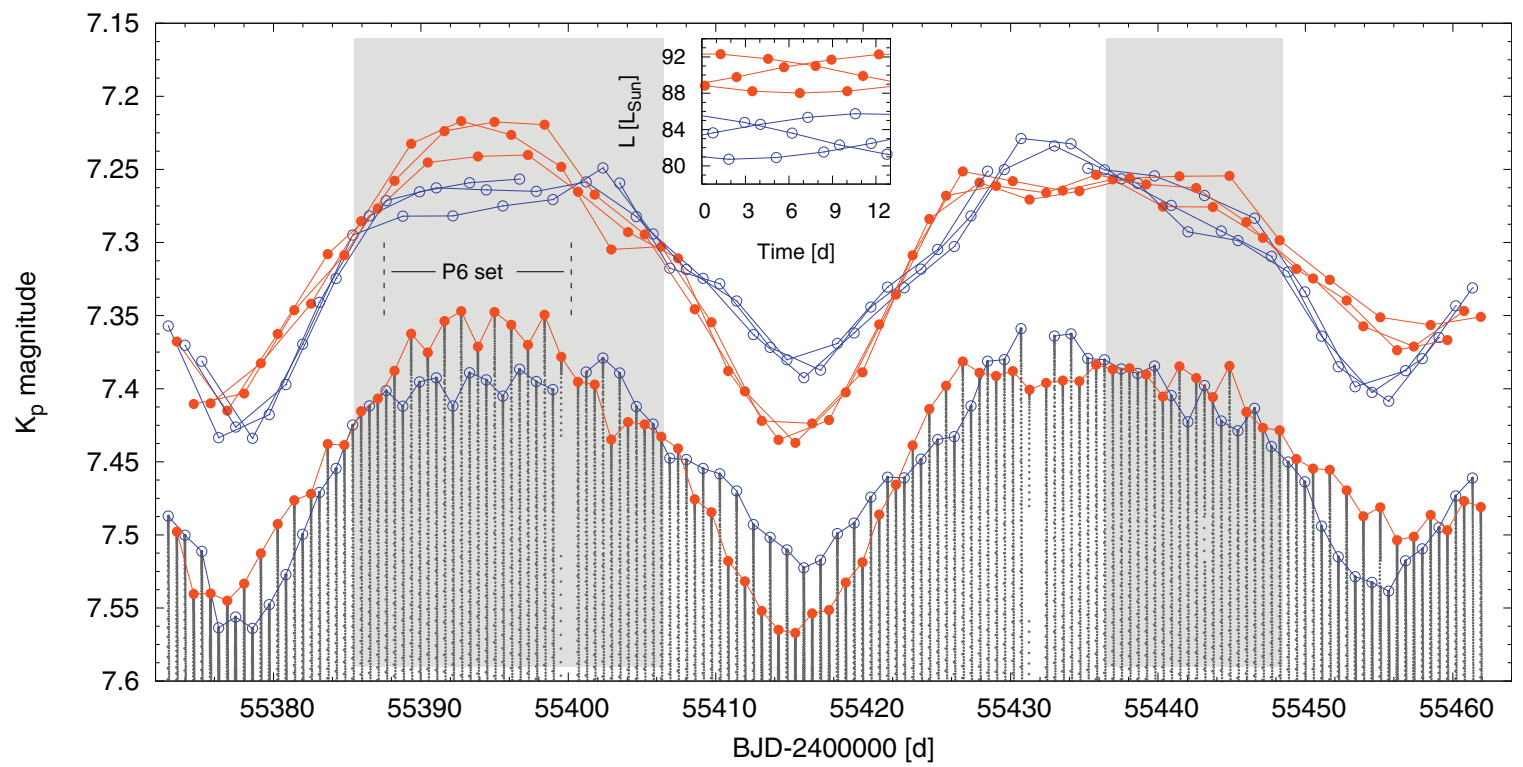

Figure 1. Short cadence Q6 data of RR Lyrae. Light gray points are the brighter end of the light curve. Black and dark gray (blue and orange in the color version) lines connect the maxima of even and odd pulsation cycles, respectively. In the case of the period-six visualization (shifted by 0.15 mag), every sixth maxima are connected, such as black/blue lines connect the $6 k, 6 k+2$ and $6 k+4$ points (circles), while gray/orange lines connect the $6 k+1,6 k+3$ and $6 k+5$ points (dots) where $k=0,1,2 \ldots$ The period-six-like sections are indicated with the gray boxes: we identified two occurrences between 55385.3-55406.3 and 55436.9-55448.3. Dashed lines mark the P6 set which we analyzed separately (see Figure 3). The inset shows the calculated luminosity of a model solution close to the 3:4 resonance. Note the similarity of the crossings between the individual blue and orange lines or branches. Model parameters $\left(\alpha_{v}\right.$ is the eddy viscosity parameter) are $M=0.64 M_{\odot}$, $L=45 L_{\odot}, T_{\text {eff }}=6500 \mathrm{~K}, \alpha_{\nu}=0.0175$. The inset covers the same time span as the P6 section.

(A color version of this figure is available in the online journal.)

against each other, e.g., the maximum values of the cycles of a (multi)periodic signal. On a return map, single-mode oscillation is represented by a single dot (no change), period doubling by two dots (small and large cycles), and double-mode pulsation is either a discrete number of points or a continuous loop, depending on whether the period ratio is commensurate or not. Return maps can provide an easier way to detect these features than conventional Fourier transforms (FTs) as FTs of RR Lyrae variations are dominated by the harmonic peaks of the main period and may contain many linear combination peaks.

In the case of Blazhko variables, however, the modulation will dominate over almost any other feature. So, instead of creating a return map, we simply plotted the maxima of even and odd pulsation cycles respectively (blue and orange lines in Figure 1) in the light curve against time. If the period doubling is constant, the two lines should never cross each other.

However, the pulsation of RR Lyr is much more complicated. The period doubling is omnipresent, contrary to the first implications from the long cadence (LC) data (Szabó et al. 2010), but it is clearly not constant. The most curious phenomenon occurscoincidentally - in Q6, the sixth quarter of Kepler observations, between BJD 55388 and 55400 (we refer to this section of the light curve as the P6 set; see Figure 1). An up-down bounce in each arm of period doubling is evident, indicating a possible additional bifurcation. Only if we connect every sixth maxima instead of every second can we smooth out most of the variations between successive maxima. Here, the fundamental mode shows "period-six" behavior, i.e., a pattern of six cycles instead of two seems to be repeating. We found one more possible case and an extension to the P6 set where the pulsation temporarily shows traces of period-six behavior, indicated by the gray boxes in Figure 1. Outside the boxes, both visualizations (every second or sixth maxima) are dominated by period doubling, confirming the temporal nature of the period-six phenomenon. Reanalysis of the Q1-Q2 data suggests period-six pattern at the second half of Q1 and the beginning of Q2 but the gap between the quarters hampers the detection.

The period-six behavior can originate from a resonance if two modes are involved in the variation. A resonance with the first overtone with a period ratio of 3:4 (0.75) would be plausible for example, compared to the period ratio range of doublemode RR Lyrae stars (Petersen 1973). We note in passing that it would be more correct to describe this resonance as 6:8 as the presence of the period doubling requires six pulsation cycles to repeat the pattern. Another possible mechanism is a periodic window in a chaotic regime. A period bifurcation cascade was indeed followed up to period-eight solutions in Kolláth et al. (2011), but we have not yet found models where the perioddoubling instability alone reached chaos. It is much more likely that the period-six behavior is connected to a resonance between the period-doubled fundamental mode and another overtone (the configuration itself can be a periodic window of stability between three-mode chaotic solutions).

\subsection{The Presence of the First Radial Overtone in RR Lyrae}

The analysis of the Q1-Q2 data of RR Lyr did not reveal any additional peaks beyond the half-integer frequencies (Kolenberg et al. 2011). The sign of the ninth overtone, locked in the 9:2 resonance, was detected in those data as an excess at $9 / 2 f_{0}$ (Szabó et al. 2010), and it is present in the Q5-Q6 short cadence data set too (Figure 2).

However, at least two significant peaks are also detectable in the expected range of the first overtone in the Q5-Q6 data, at $f=2.327$ and $2.342 \mathrm{day}^{-1}$, with amplitude ratios $\left(A_{0} / A_{1}\right.$, the ratio comparing the amplitude of the $f_{0}$ peak of the fundamental mode to the $f_{1}$ peak(s)) being 155 and 170, respectively. The presence of multiple peaks indicate amplitude variations and quite possibly phase variations as well. If analyzed separately, the Q5 and Q6 data result in somewhat different values, with 


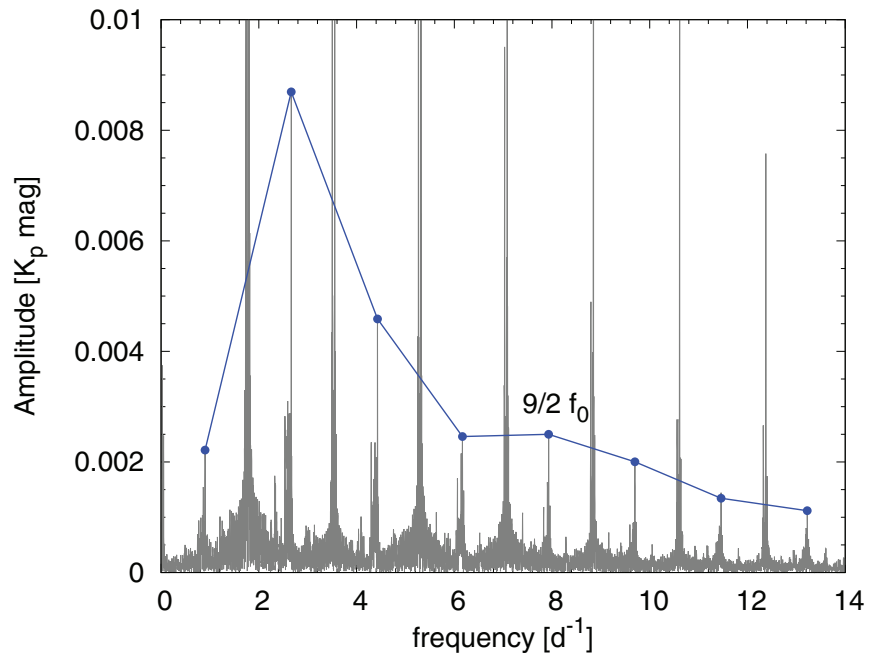

Figure 2. Amplitudes of the highest half-integer peaks at every $(2 n+1) / 2 f_{0}$ value (blue points). The Fourier spectrum of the Q5-Q6 data, prewhitened with the $n f_{0}$ peaks, is shown in gray. There is an excess at $9 / 2 f_{0}$, at the position of the ninth overtone, similar to Figure 8 in Szabó et al. (2010).

(A color version of this figure is available in the online journal.)

$A_{0} / A_{1}=138$ and 102 for a sole significant peak. The spectrum of the P6 set (lower panel in Figure 3) shows a stronger signal with $A_{0} / A_{1}=67$. The amplitudes are limited to a few millimagnitudes in all subsets, but thanks to the photometric precision, the individual peaks have signal-to-noise ratios of at least 4-6, the lowest values corresponding to the spectrum of the Q5 data only, where we did not detect period-six-like stages.

Period ratios (or $f_{0} / f_{1}$ frequency ratios) vary between 0.753 and 0.758 for the different subsets and/or peaks: For the P6 set it is 0.756 . These ratios are close to, but not exactly 0.750 , the value corresponding to a 3:4 resonance. The period ratio difference might arise from the uncertainty in the frequency determination, either caused by the frequency variations connected to the Blazhko effect or, in the case of the P6 set, the shortness of the data set. But it is more likely that the system temporarily approaches the vicinity of the resonance and the frequency difference reflects the distance from it. An additional mode with the same ratio (0.754) was detected in UZ Vir, too (Sódor et al. 2012).

This is the first time that three radial modes have been unambiguously detected in an RR Lyrae star: the fundamental, the first overtone, and the third being the ninth overtone, which is responsible for the period doubling. The detection is also strongly supported by the nonlinear models, described below. It is also clear however that the inclusion of period doubling and the first overtone still does not describe the light variations of RR Lyr perfectly. The two branches of the period doubling cross each other and reverse, with intervals ranging from a few pulsation periods to about 30 days, which is close to an entire Blazhko period ( $\sim 39$ days). These variations could be explained by yet another physically relevant frequency close to the halfinteger peaks (as it modulates the period doubling), or could be internal variability, as found in BL Herculis models (Smolec \& Moskalik 2012).

\section{MODEL CALCULATIONS}

The modulations and possible bifurcations in period doubling raise further questions regarding the dynamics of the system. To study the possible processes that might be responsible
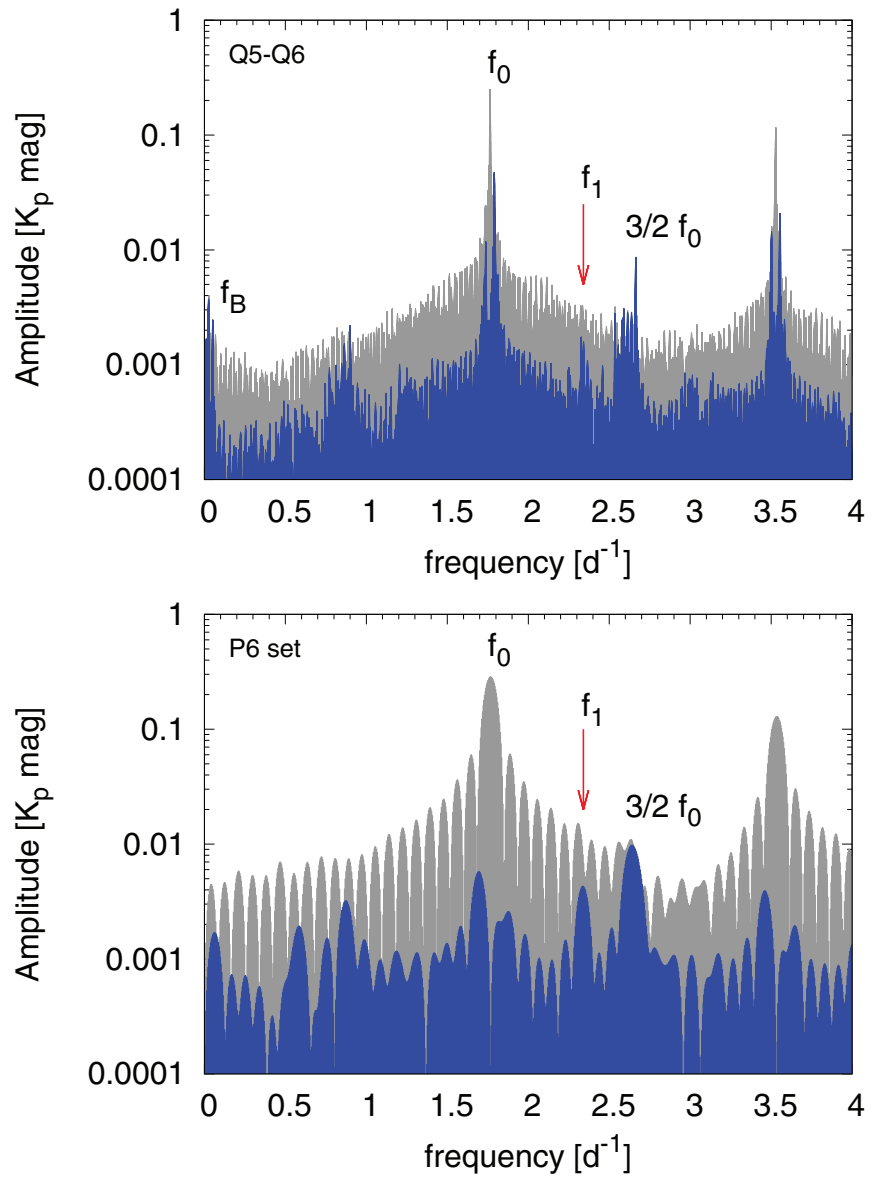

Figure 3. Detection of the first overtone $\left(f_{1}\right)$ in the Kepler measurements of RR Lyr. The original spectra are in gray and the spectra prewhitened with the main frequency $\left(f_{0}\right)$ and its harmonics $\left(n f_{0}\right)$ are in black (blue in the color version). The upper panel shows the entire Q5-Q6 data while the lower panel shows only the P6 set from Q6 (see Figure 1). A forest of peaks is evident at $3 / 2 f_{0}$ in the upper panel, indicating the strong variability of the period doubling. (A color version of this figure is available in the online journal.)

for this dynamical behavior, we conducted a broad nonlinear model survey with the Florida-Budapest turbulent convective hydrodynamic code (Kolláth \& Buchler 2001; Kolláth et al. 2002). The results of this survey will be published in a subsequent paper (Z. Kolláth et al., in preparation); here we only summarize the relevant facts.

We found that period doubling changes the stability of the fundamental mode. The mode in itself is stable against the perturbations of the first overtone, but unstable against the ninth overtone and the period-doubling bifurcation occurs. The period-doubled mode is, however, unstable (or at least marginally stable) against the perturbations of the first overtone. The result is a new pulsation state that looks like a new kind of double mode behavior but is in fact created by the interaction of three different radial modes. In some of the models, this twoperiod state bifurcates further to resonant or chaotic solutions. Detailed properties of these models, their occurrence along the instability strip will be investigated in forthcoming papers. We note that the two chaotic model solutions that were analyzed by Plachy et al. (2012) turned out to be three-mode solutions as well.

The various dynamical states are best represented with return maps. In case of simple period doubling, we will have two dots, corresponding to the lower and higher amplitude cycles. If two 


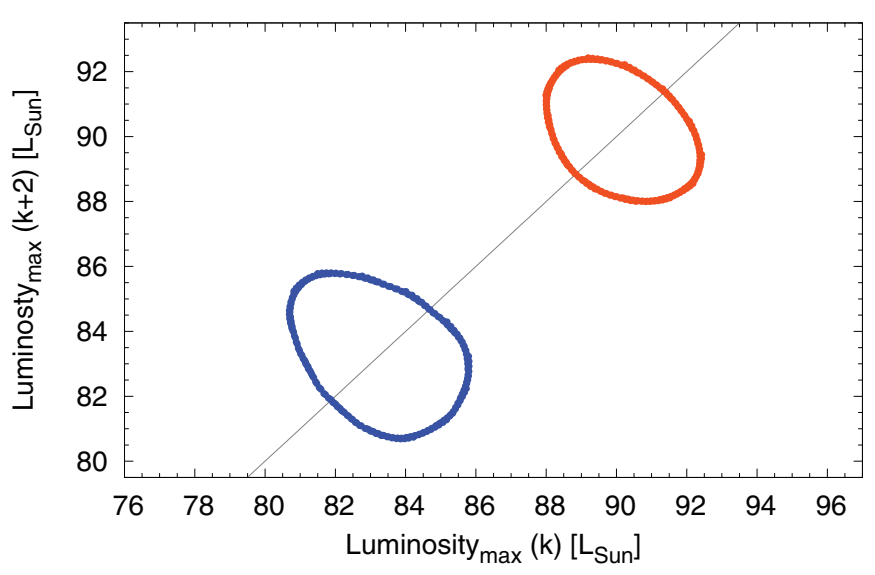

Figure 4. First return map of a three-mode model. The figure shows the maxima of the luminosity variations plotted against each other. Here, we show the maxima of the $k$ th vs. the $(k+2)$ th cycles instead of successive ones to separate the smaller and larger cycles of period doubling. Although three radial modes are present, the ninth overtone is locked in a resonance with the fundamental mode, and it is only detectable through the presence of period doubling, hence we observe two loops instead of a single one, both representing the double-mode pulsation between incommensurate $f_{0}$ and $f_{1}$ frequencies. Model parameters are the same as in Figure 1.

(A color version of this figure is available in the online journal.)

different modes are present, the return map will either show a set of points or a loop, depending on the phase relations (e.g., are the two modes in resonance or not).

Take for example the two loops in Figure 4 which is the same model as in the inset in Figure 1. The presence of a loop at lower values and another at higher values indicate that both period doubling and an additional periodicity is present in this system, a state that resembles the Kepler observations of RR Lyrae.

\subsection{Comparison with the Models}

The detection of three different radial modes in an RR Lyrae star may allow for nonlinear asteroseismic studies: comparison of the observations not only with linear pulsation periods but also with mode amplitudes, resonances and frequency shifts.

Our first calculations focused on the possible 3:4 resonance. The corresponding 0.750 period ratio, and especially the exact observed period ratios in RR Lyr between the first overtone and the fundamental mode are a challenge: They lie at the edge of the accessible range for the classical double-mode pulsation in the Petersen diagram (see Figure 6 in Szabó et al. 2004). We have not succeeded in finding models with an exact 3:4 resonance yet, though we found resonant solutions with higher integers corresponding to lower period ratios. These models can help us identify possible (near-)resonances between the fundamental mode and the first overtone in other RR Lyrae stars.

However, models with a linear period ratio of 0.747 instead of 0.750 do in fact approach the vicinity of the 3:4 resonance. These calculations indicate that RR Lyr only approaches the resonance during the period-six phases but does not lock into it. Although the models favor solutions below the 0.750 frequency ratio instead of above (as in the case of RR Lyr), the topology, like the distances and crossings of the six branches are quite similar, especially in the P6 section (see inset in Figure 1) and support our findings. Swapping of either three branches simultaneously was not observed in the models, confirming that yet another mechanism must be behind some of the remaining features, like the modulation of the period doubling.
We also note that the difference between linear and nonlinear period ratios in the models approaches 0.003 and sometimes (especially in resonant states) up to 0.005 . This nonlinear shift is comparable to the observed period ratio range of the Petersen diagram of the normal double-mode RR Lyrae (RRd) stars.

\section{SUMMARY}

The detection of so many interesting features in a star of long observational history remarkably illustrates the advantages of space-based photometry. A decade ago, Smith et al. (2003) noted that the residuals after fitting the primary components of the spectrum (the triplet at the fundamental mode, their harmonics and the modulation frequency) exceeded the expected observational error. The first observations of Kepler revealed the period-doubling phenomenon (Kolenberg et al. 2010; Szabó et al. 2010) and now the first overtone was also detected in the short cadence data. The results and implications of the analysis can be summarized as follows.

1. Three different radial modes were detected in RR Lyr, the eponym of its class: the fundamental mode and the first and ninth overtone. However, the ninth overtone is locked in a resonance with the fundamental mode and can be detected only through the presence of period doubling (Kolláth et al. 2011), causing the observed variations to resemble doublemode behavior.

2. The first overtone has a very small amplitude (few millimagnitudes), in contrast with the fundamental mode or the classical double-mode RRd stars, explaining the nondetection from the ground.

3. A state resembling period-six bifurcation was detected in the light variations of RR Lyr. This behavior is most likely caused when the system approaches the vicinity of a $3: 4$ resonance between the fundamental mode and the first overtone.

4. We have been able to reproduce the three-mode state in nonlinear hydrodynamic models. Although we have not been able to model the $3: 4$ resonance or the higher than 0.750 period ratio of RR Lyr, the amplitude ratios can be reproduced and higher-order resonances have been found as well. Furthermore, near-resonance models below the 0.750 value have very similar characteristics, raising hopes for applying nonlinear asteroseismology to Blazhko RR Lyrae stars.

5. Even the inclusion of three different modes cannot explain all the variations we see in the period doubling, indicating that the pulsation of RR Lyrae stars may be even more complex.

Our radial nonlinear hydrodynamic calculations demonstrated that the period-doubled fundamental mode may lose its stability with respect to the first radial overtone. It then is straightforward to expect similar nonlinear destabilization with respect to low order non-radial modes as well. This process might provide the background for the fact that additional pulsation frequencies occur more frequently in stars where period doubling is also detected. First overtone signals were identified in three other stars in the Kepler sample by Benkő et al. (2010), and the case of RR Lyr suggests that more may be discovered. The period ratios of peaks that can be plausibly associated with the first overtone are for V354 Lyr (KIC 6183128): $f_{1} / f_{0}=0.729$, and for V360 Lyr (KIC 9697825): $f_{1} / f_{0}=0.721$. For V445 Lyr (KIC 6186029), Benkő et al. 
(2010) noted a very complex pattern of peaks while the detailed analysis of Guggenberger et al. (2012) concluded that among the additional frequencies, two can be found at ratios $f_{N} / f_{0}=0.703$ and $f_{0} / f_{1}=0.730$. Even more stars show the signature of the second overtone for which Molnár et al. (2012) already proposed the alternate idea of a three-mode resonance, where the equation $3 f_{0}+f_{2}=f_{9}$ may describe the relation between the three frequencies.

The results indicate that interactions between radial and non-radial modes may indeed play a more crucial role in the pulsation of stars. Buchler \& Kolláth (2011) showed that the resonance responsible for period doubling can cause Blazhkolike modulation in amplitude equations. Nonlinear asteroseismology promises to understand these new findings, and might even help unlock the mystery of the Blazhko effect itself.

Funding for this Discovery Mission is provided by NASA's Science Mission Directorate. The Kepler Team and the Kepler Guest Observer Office are recognized for helping to make the mission and these data possible. This work was supported by the Hungarian OTKA grants K83790, K81421, and MB08C 81013, as well as the "Lendület" programme, and the European Community's Seventh Framework Programme (FP7/2007-2013) under grant agreement No. 269194. R.Sz. acknowledges the Bolyai János Scholarship of the Hungarian Academy of Sciences. K.K. is supported by a Marie Curie International Outgoing Fellowship within the 7th European Community Framework Programme. R.Sz. and K.K. warmly thank the KITP staff of UCSB for their hospitality during the "Asteroseismology in the Space Age" programme. This research was supported in part by the National Science Foundation under grant No. NSF PHY05-51164.

Facility: Kepler

\section{REFERENCES}

Benkő, J. M., Kolenberg, K., Szabó, R., et al. 2010, MNRAS, 409, 1585

Borucki, W. J., Koch, D., Basri, G., et al. 2010, Science, 327, 977

Buchler, J. R., \& Kolláth, Z. 2011, ApJ, 731, 24

Chadid, M., Benkő, J. M., Szabó, R., et al. 2010, A\&A, 510, 39

Gilliland, R. L., Brown, T. M., Christensen-Dalsgaard, J., et al. 2010, PASP, 122,131

Guggenberger, E., Kolenberg, K., Chapellier, E., et al. 2011, MNRAS, 415, 1577

Guggenberger, E., Kolenberg, K., Nemec, J. M., et al. 2012, MNRAS, 424, 649

Kolenberg, K., Bryson, S., Szabó, R., et al. 2011, MNRAS, 411, 878

Kolenberg, K., Szabó, R., Kurtz, D., et al. 2010, ApJ, 713, L198

Kolláth, Z., \& Buchler, J. R. 2001, in Stellar Pulsation-Nonlinear Studies, ed. M. Takeuti \& D. D. Sasselov (Astrophysics and Space Science Library Series, Vol. 257; Dordrecht: Kluwer), 29

Kolláth, Z., Buchler, J. R., Szabó, R., \& Csubry, Z. 2002, A\&A, 385, 932

Kolláth, Z., Molnár, L., \& Szabó, R. 2011, MNRAS, 414, 1111

Molnár, L., Kolláth, Z., \& Szabó, R. 2012, ASSP, in press (arXiv:1201.5628v1)

Nemec, J. M, Smolec, R., Benkő, J. M., et al. 2011, MNRAS, 417, 1022

Petersen, J. O. 1973, A\&A, 27, 89

Plachy, E., Kolláth, Z., \& Molnár, L. 2012, ASSP, in press (arXiv:1202.0271v1)

Poretti, E., Paparó, M., Deleuil, M., et al. 2010, A\&A, 520, 108

Smith, H., Church, J. A., Fournier, J., et al. 2003, PASP, 115, 43

Smolec, R., \& Moskalik, P. 2012, MNRAS, in press (arXiv:1207.2151v1)

Smolec, R., Moskalik, P., Kolenberg, K., et al. 2011, MNRAS, 414, 2950

Sódor, Á., Hajdu, G., Jurcsik, J., et al. 2012, MNRAS, in press (arXiv:1208.0139v1)

Szabó, R., Kolláth, Z., \& Buchler, J. R. 2004, A\&A, 425, 627

Szabó, R., Kolláth, Z., Molnár, L., et al. 2010, MNRAS, 409, 1244 